\title{
Interpersonal Relationships in Sport Groups
}

Philipp Sauer, Ed.D.

United States Sports Academy, Germany

\begin{abstract}
The paper presents an overview of the unique structure and complexity of sport teams. It provides the reader with general and basic information about both, the physiological and psychological structure of sport teams. It describes the unique characteristics of interpersonal relationships in sport teams, and provides information on how individual capabilities and motivational processes might influence group performance. In this context, particular attention is paid to specific models that examine group processes and interaction. In addition, it illustrates the consequences of social influence on group interaction and performance. Finally, the paper provides an outlook to future tendencies of group dynamics and the importance of interpersonal relationships in sport groups.
\end{abstract}

Keywords: group dynamics, group interaction, social influence, interpersonal relationship, group processes, sport team, group cohesion, performance

\section{INTRODUCTION}

Over the years, head coaches, managers, and other persons in charge, have learned that it is not only the physiological, but especially the psychological structure of a sports team that needs to be monitored constantly. "The concept of group structure describes the different patterns of relationships that exist in groups and helps us to identify the features common to them all" [Shaw, Gorely, \& Corban, 2005, p. 225). In this context, Horn (2008, p. 215) cites Carron, Hausenblas, and Eys (2005), who defined a sports team "as a collective of two or more individuals who possess a common identity, have consensus on a shared purpose, share a common fate, exhibit structured patterns of interaction and communication, hold common perceptions about group structure, are personally and instrumentally interdependent, reciprocate interpersonal attraction, and consider themselves to be a group".

Basically, the structure of a sport group is classified into its physical structure and its psychological structure. The physical structure describes the whole organization, and composition of a group. It includes the leadership hierarchy of the sports group, but also the complete administrative and formal effort. In instances like organized sport teams, "individuals come into a situation where the specific organized structure is is in place" (Carron \& Eys, 2012, p. 152). The nature of the sport has usually established through rules, while other factors like the organization and administration of the team has evolved through traditions and the team's culture. In the past, research of the physical structure in sport teams mainly focused on the individual capabilities of its team members. However, sports history has proven, that the performance of a team cannot be measured by summing up the individual abilities of each player. While the physical structure of a sports team consists of variables that can be controlled and held constant, - like team size or rules - the psychological structure of a team contains several variables that need to be monitored constantly to ensure maximum group performance.

Every coach and team manager intends to create a team culture, which promotes positive energy and provides team members with a comfortable feeling. Finally, it is the team's psychological structure that influences each aspect of the team's atmosphere and experience. Interaction and communication are basic processes in every group. "With that interaction and communication, differentiation among individuals appears, and the four components that most clearly reflect the presence of a psychological structure emerge" (Carron \& Eys, 2012, p. 152). Those four components of a psychological structure in sport teams are group position, group status, group role, and group norms. 


\section{REVIEW OF LITERATURE}

\subsection{Interpersonal Relationships in Sport Groups}

Interpersonal relationships are a critical factor in every kind of group. According to McGrath (1984, p.7) "groups are those social aggregates that involve mutual awareness and potential interaction". Therefore, asking questions about the importance and effect of interpersonal relationships in sports groups is logical.

\subsection{Concepts and Models}

In McGrath's conceptual framework for groups (1984), interaction is defined as the central factor in group processes and dynamics. According to this framework, "individual characteristics influence group structures and patterns, environmental properties affect the group task and situation, and those factors collectively influence the behavioral setting under which group interaction takes place" (Gill \& Williams, 2008, p. 242). In turn, the interaction within the group might be influenced by all those elements mentioned-above, or by the interaction itself.

A good example, which illustrates this framework, can be found in a soccer team. Every player of the team has individual characteristics, like skills, motivation, experience, etc. Those characteristics automatically affect the group structure and its attributes like positions, statuses, leaders, etc. Positions and strategies might also be influenced by environmental factors, such as weather, the opponent, and the audience. "The ongoing interaction and the game progress may then change the environment, individuals, and relationships" (Gill \& Williams, 2008, p. 242). A player who made several serious mistakes might loose confidence, and will not be able to fulfill the expectation of the coach and his teammates. This would probably lead to a substitution and maybe to a change of the strategy.

In addition to McGrath's conceptual framework, Widmeyer, Brawley, \& Carron (2002) have developed a linear model that defines individual characteristics and environmental factors as starting input components for group dynamics. These starting components influence group structure, which in turn influences group cohesion, which then influences group processes, which finally influence individual and group outputs (Gill \& Williams, 2008, p. 243). This framework highlights the importance and prominence of group cohesion. In 2005, Carron modified this framework, by moving cohesion to the output side of the model, in order to reflect the mutual relationships and dynamic nature of group processes and individual outputs (Gill \& Williams, 2008). The modified model classifies four categories of cohesion: situational, personal, leadership, and team (Horn, 2008). "The four aspects of group cohesion specified by the conceptual model are related to both the antecedents and the consequences of cohesiveness in sport" (Horn, 2008, p. 229).

\subsection{Group Performance}

Every head coach or manager of a sports team intends to maximize group performance. One of the most famous and accepted maxims is that the best players make the best team. However, sports history has shown that simply summing up the individual abilities of team members does not maximize group performance, as a champion team would rather defeat a team of individual champions. Understanding the group process-interaction is of outmost importance in order to describe group performance.

In this context, Steiner (1972) proposed a simple model, which illustrates the relationship between individual and group performance. The model describes a group's actual productivity as the result of its potential productivity less losses due to faulty processes. The potential productivity refers to the best possible performance of the team by considering all resources available. Those resources mainly describe all relevant knowledge and skills of individual members, including the overall level and distribution of talents (Gill \& Williams, 2008). The most important resource of any sports team can be identified in the athlete's individual abilities.

Processes within a sports team describe both, individual and interactive processes, which are used in order to achieve common goals. Steiner classifies process losses into two categories:

- Coordination losses describe failure in strategy and poor timing. A good example is a soccer team, which fails to get the ball to their top scorer (Gill \& Williams, 2008).

- Motivational losses mainly occur as a result of a lack of self-efficacy and collective efficacy. A typical situation, in which motivational losses occur can be found when a soccer team gets trailed 
by two or more goals in a short period of time. Such situation usually decreases motivation among team members, and requires a change of the strategy.

The main task of a head coach is to find effective ways to reduce those faulty processes. $\mathrm{He} / \mathrm{she}$ has to develop organizational strategies for being able to reduce coordination losses and keeping motivation high even in difficult situations.

Research on the role of group processes revealed a phenomenon that is called the Ringelmann-effect, "whereby as the group size increases, the individual productivity of the people in the group decreases, by as much as $50 \%$ in some cases" (Barker et al., 2007, p. 339). The larger the group gets, athletes experience a feeling of not being accountable for their own performance, as they can "hide" behind the other athletes. Research assumes that the Ringelmann effect is rather caused by motivational losses than by coordination losses.

Latane, Williams, \& Harkins (1979) called those motivational losses in teams "social loafing". Motivational losses are most evident when individual contributions of team members might not be identified or seem to be dispensable (Barker et al., 2007). Athletes who display social loafing usually have a lack of self-efficacy and doubt if their actions can contribute to the team's success. In contrast to this, research found out that social loafing was eliminated, when team members believed their individual outputs were identifiable.

\subsection{Social Support}

Another ongoing interaction process of sport teams is social support. Shumaker \& Brownell (1984, p. 13) define social support as "an exchange of resources between at least two individuals perceived by the provider or the recipient to be intended to enhance the well-being of the recipient". According to Brewer (2009, p.76) "social support is the coping resource that has been tied most frequently to sport injury". However, social support also plays a major role in important group dynamic issues, like group cohesion. Gill \& Williams (2008) cite Rosenfeld and Richmann (1997), who developed a model that includes three broad types of social support. Those three types involve direct assistance (tangible support), advice (informational support), or encouragement (emotional support) (Gill \& Williams, 2008). Social support might help athletes to improve self-efficacy and provide them with a feeling of security and belonging. Especially, athletes who suffer a serious injury experience a cognitively, emotionally, and behaviorally challenging situation (Loutsch, 2007). During such a hard time, it can be very helpful for an injured athlete to experience feelings of support and appraisal. A good example, which illustrates how sport clubs might provide social support to their athletes, is an early extension of the contract of an injured athlete. A few years ago, the German soccer club FC Bayern Munich untimely extended the contract of a player who was injured for more than one year. Such action includes all three types of social support, as this action not only provides tangible support, but also motivational and emotional support.

\section{SUMMARY AND OUTLOOK}

As mentioned previously, every head coach and sports manager intends to maximize performance of his/her team. A basic understanding of the team's structure and processes is a prerequisite for being able to maximize performance. In the recent past, the importance of psychological factors in sports teams has continuously increased. The history of sports provides numerous examples of athletes who were not able to trigger their full potential under certain circumstances. Therefore, the significance of individual assistance for athletes has continuously increased. Sports psychologists and head coaches have to pay more attention on the effects of interpersonal relationships, like the influence of turnover of team members. To enhance group motivation and performance, head coaches have to consider and treat each athlete as unique individuals. To trigger an athlete's full performance requires the club and the whole leading staff to find out something special about each athlete and treat them as individually as possible (Gill \& Williams, 2008).

Interpersonal relationships and group processes have become key factors to fully understand group dynamics in sport teams. "Coaches, instructors, and leaders might direct efforts toward developing interactive skills, as well as performance skills in order to reduce coordination losses and enhance group performance" (Gill \& Williams, 2008, p.264). In this context, finding ways and strategies to reduce motivational losses will also become more important. This could mean that the individual roles and contributions of team members are clearly identified and evaluated. 
The past Olympic Summer Games in Rio, once again showed that many athletes were not able to deal with pressure and high expectations, but failed to retrieve their potential. However, the Summer Games also revealed that many Olympic Sports Confederations have enforced psychological assistance and observation. The German Olympic Sports Confederation recently announced that it aims to expand the psychological field, not only to improve an athlete's performance, but also the athlete's personality and health.

However, learning more about the dynamics and relationships of sport teams is very challenging. Sport psychologists and researchers have to focus on relationships between several variables. They have to analyze potential variables, like gender, cultural background, or other types of influence in order to improve team effectiveness. They have to consider that "it is not only understood that each individual on the team be capable of performing well at the sport, but that the entire team have an overall sense of efficacy when it comes to the task at hand" (Manning, 2007, p.68).

\section{Conclusion}

This paper provided an overview of the complexity and importance of the structure of sport groups. The paper focused on the nature and characteristics of interpersonal relationships. It used McGrath's conceptual framework to illustrate the significance of interaction as the central factor in group processes and dynamics. It has pointed out that "individual characteristics influence group structures and patterns, environmental properties affect the group task and situation, and those factors collectively influence the behavioral setting under which group interaction takes place" (Gill \& Williams, 2008, p. 242). "Coaches should remain cognizant of the larger finding, namely, that trying to focus on one factor may impact the overall success of the team, because of how each of these factors are interrelated and contribute to the overall successfulness or unsuccessfulness of the team" (Manning, 2007, p.69). In addition, the linear model by Widmeyer, Brawley, \& Carron (2002) illustrated the relevance and prominence of group cohesion. It also classified four major correlates to group cohesion: situational, personal, leadership, and team.

The paper analyzed the relationship between individual and group performance. In this context, the paper made use of Steiner's model that describes a group's actual productivity as the result of its potential productivity less losses due to faulty processes. Those losses were classified into coordination losses and motivational losses. Therefore, improving group performance is unmistakably related to the reduction of faulty processes. Coaches must find ways and establish strategies to identify and eliminate any faulty process within a team.

Finally, triggering a sport group's best possible performance heavily depends upon the head coaches' ability to assess the interrelationships of various factors. It is not sufficient to focus on the relationships between different role elements, but a head coach needs to understand the full dimension of numerous influential factors on group dynamics.

\section{REFERENCES}

[1] Shaw, D., Gorely, T., \& Corban, R. (2005).Sport \& exercise psychology. Independence, KY: Taylor \& Francis.

[2] Horn, T. S. (2008). Advances in sport psychology (3rd ed.). Champaign, IL: Human Kinetics.

[3] Carron, A. V., \& Eys, M. A. (2012). Group dynamics in sport (4th ed.). Morgantown, WV: Fitness Information Technology.

[4] McGrath, J. E. (1984). Groups: Interaction and performance. Englewood Cliffs, NJ: Prentice Hall.

[5] Gill, D. L., \& Williams, L. (2008). Psychological Dynamics of Sport and Exercise (3rd ed.). Champaign, IL: Human Kinetics.

[6] Widmeyer, W. N., Brawley, L. R., \& Carron, A. V. (2002).Group dynamics in sport. In T. S. Horn (Ed.), Advances in sport psychology (2nd ed., pp. 285-308). Champaign, IL: Human Kinetics.

[7] Steiner, I. D. (1972). Group process and productivity. New York, NY: Academic Press.

[8] Barker, R., Gledhill, A., Lydon, C., Miles, A., Mulligan, C., Saffery, G., Sutton, L. (2007). BTEC National Sport, Book 1 (2nd ed.). Oxford: Heinemann.

[9] Latane, B., Williams, K. D., \& Harkins, S. G. (1979). Many hands make light the work: The causes and consequences of social loafing. Journal of Personality and Social Psychology, 37(), 823-832. 
[10] Shumaker, S. A., \& Brownell, A. (1984). Toward a theory of social support: Closing conceptual gaps. Journal of Social Issues, 40(), 11-36.

[11] Brewer, B. W. (Ed.). (2009). Sport psychology. Hoboken, NJ: International Olympic Committee.

[12] Loutsch, J. A. (2007). Perceived social support systems during athletic injury recovery in collegiate club sport athletes (Masters thesis). Retrieved from http://books.google.de/books? $\mathrm{id}=6 \mathrm{dhPs} 8 \mathrm{dbipUC} \&$ printsec $=$ frontcover $\# \mathrm{v}=$ onepage $\& \mathrm{q} \& \mathrm{f}=$ false

[13] Manning, C. T. (2007). Relationship among team collective efficacy, cohesion, and coaching competency in sports (Doctoral dissertation, Utah State University). Retrieved from http://books. google.de/books?id=NemVNYsJPFkC\&printsec=frontcover\#v=onepage\&q\&f=false 